\title{
Incidence and outcome of rhabdomyolysis after type A Aortic Dissection surgery -a retrospective analysis
}

\author{
PRAVEEN SIVADASAN ${ }^{1}$, Amr Omar², Cornelia Carr ${ }^{2}$, Abdul Rasheed Pattath ${ }^{1}$, Samy \\ Hanoura $^{1}$, Suraj Sudarsanan ${ }^{1}$, Hany Ragab ${ }^{1}$, Hatim Sarhan ${ }^{2}$, Arunabha Karmakar ${ }^{1}$, and \\ Rajvir Singh ${ }^{2}$ \\ ${ }^{1}$ Hamad Medical Corporation \\ ${ }^{2}$ Hamad Medical Corp
}

June 22, 2021

\begin{abstract}
Objectives: To study the incidence of rhabdomyolysis (RML) after ascending aortic dissection surgery and to correlate with the outcome especially in terms of renal function. The secondary objective was to pinpoint the perioperative risk factors associated with the development of Rhabdomyolysis and adverse renal outcome after Aortic dissection repair Design: Retrospective singlecenter study Settings: Tertiary cardiac center Participants: Patients who had undergone ascending aortic dissection repair from 2011-2017. Interventions: Two groups Group 1- with RML creatine kinase (CK) above cut-off levels 2500 U/Liter and Group 2 without RML. The potential determinants of RML and the impact of the same on the patient outcome; especially on postoperative renal function was studied. Measurements and main results: Out of 33 patients studied, 21 patients (64\%) developed Rhabdomyolysis (Group RML) and 12 did not (Group non-RML). Demographic and intraoperative factors had no significant impact on the incidence of RML. There was a significantly higher incidence of AKI in the RML group (90\%) than in the non-RML group (25\%). All 4 patients who went into new post-operative dialysis belonged to the RML group. The peak troponin levels were significantly higher in RML group Conclusion: In this study, we found a high incidence of RML after aortic dissection surgery which paralleled with an adverse renal outcome and need for postoperative dialysis. Identification of risk factors and early intervention might help to mitigate the severity of renal failure. Further large-scale prospective trials are warranted to investigate the predisposing factors and influence of RML on outcome
\end{abstract}

\section{TITLE PAGE}

Incidence and outcome of rhabdomyolysis after type A Aortic Dissection surgery -a retrospective analysis

Praveen C Sivadasan ${ }^{1} *$ Email: drpraveencs@gmail.com Amr S Omar ${ }^{1.2,3}$ Email: a_S_omar@yahoo.com Cornelia S Carr ${ }^{1}$ Email: ccarr@hamad.qa Abdul Rasheed A Pattath ${ }^{1,3}$ Email: drrashmanson@gmail.com Samy Hanoura ${ }^{1,3,4}$ Email: sehanoura73@yahoo.com Suraj Sudarsanan ${ }^{1}$ Email: drsurajsudarsan@gmail.com Hany Ragab ${ }^{1}$ Email: Hanyragab73@gmail.com Hatem Sarhan ${ }^{1,5}$ Email: HSarhan2@hamad.qa Arunabha Karmakar ${ }^{1,5}$ Email: arunabha.karmakar@gmail.com

Rajvir Singh ${ }^{5}$

Email: rsingh@hamad.qa

1. Department of Cardiothoracic Surgery/Cardiac Anaesthesia \& ICU Section, Heart Hospital, Hamad Medical Corporation, Doha, (PO Box: 3050), Qatar

2. Department of Critical Care Medicine, Beni Suef University, Egypt

3. Weill Cornell Medical College-Qatar, Doha, Qatar 
4. Department of Anesthesia, Al-Azhar University, Cairo, Egypt

5. Department of Medical Education, Hamad Medical Corporation, Doha, Qatar

6. Department of Medical Research, Hamad Medical Corporation, Doha, Qatar

* Corresponding author. Department of Cardiothoracic Surgery/Cardiac Anaesthesia \& ICU Section, Heart Hospital, Hamad Medical Corporation, Doha, (PO: 3050), Qatar Email: drpraveencs@gmail.comIncidence and outcome of rhabdomyolysis after type A aortic dissection surgery. A single center retrospective analysisIntroduction

Despite ongoing research, the etiology of acute kidney injury (AKI) remains incompletely understood; especially after aortic dissection surgeries. The association of rhabdomyolysis (RML) and acute kidney injury (AKI) with cardiac surgery has been evaluated and reported by us in a recently concluded study ${ }^{1}$, but there is a lack of robust data regarding the same in aortic dissection surgeries specifically.

Rhabdomyolysis is a syndrome characterized by breakdown of skeletal muscles and release of toxic intracellular contents into the systemic circulation causing damage to renal tubules. RML may be caused by a myriad of etiologies, predominantly by direct trauma to muscles as seen in crush injuries, burns or prolonged muscle compression. It could also be associated with congenital disorders of metabolism, certain drugs like anesthetic agents, neuroleptic agents and statins, infections and sustained muscle contraction (seizures and prolonged exercise for example) ${ }^{2}$. The illness might vary in severity from asymptomatic elevations in markers of muscle injury (namely Creatinine Kinase (CK) and Myoglobin); to severe cases associated with extreme enzyme elevation and renal shut down culminating in dialysis ${ }^{3}$. Postoperative Rhabdomyolysis is being increasingly recognized as a cause of renal failure. RML is a well-known complication after bariatric, Urologic and orthopedic surgery ${ }^{4}$. One retrospective review which analyzed myoglobin as a marker of myocardial injury post-cardiac surgery reported myoglobin to be superior to creatine kinase (CK) for prediction of mortality and need for renal replacement therapy ${ }^{5}$. The literature linking RML to cardiac surgery was largely confined to isolated case reports ${ }^{6}$ until our study ${ }^{1}$ was published. The aforementioned study in our center, in which we noticed an unusually high incidence of RML among the patients undergoing type A aortic dissection repair laid the foundation for this broader retrospective analysis specifically looking for a link between RML and aortic surgeries.

Acute kidney injury (AKI) complicates recovery from cardiac surgery in up to $30 \%$ of patients and places patients at a 5-fold increased risk of death during hospitalization. Etiology is often multifactorial and preventive strategies are limited. AKI that requires renal replacement therapy occurs in $2-5 \%$ of patients following cardiac surgery and is associated with $50 \%$ mortality $^{7}$.

Aortic surgeries are specifically associated with a higher incidence of renal complications than other types of cardiac surgery (with a reported incidence of AKI ranging from $18 \%$ to $55 \%)^{8}$. A significant number of patients with ascending aortic dissection (AAD) have chronic renal impairment on presentation ${ }^{9}$. Renal failure and dialysis after aortic dissection surgery is an independent predictor of mortality as per the International Registry of Acute Aortic Dissection. Perioperative predictors for postoperative AKI and renal replacement therapy according to various studies were estimated preoperative glomerular filtration rate, coronary ischemic time, renal artery involvement in dissection, total arch replacement, preoperative oliguria, longer cardiopulmonary bypass and hypothermic circulatory arrest times, high body mass index, elevated C reactive protein, perioperative sepsis and postoperative bleeding requiring a surgical revision ${ }^{10-14}$. Till date, there are no published studies linking rhabdomyolysis with aortic dissection except for a single case report ${ }^{15}$. Myoglobin has reportedly been linked to renal morbidity and mortality after thoracic and thoraco-abdominal aortic surgeries ${ }^{16}$

\section{Patients}

In this retrospective analysis, we reviewed the charts of all patients who had undergone type A aortic dissection repair operation during the period 2011 to 2017 in a tertiary care cardiac surgical center in Qatar. We got prior approval from the Institutional review board (reference number MRC-01-18-073) and a waiver of informed consent for data collection was obtained as well. 


\section{Data collection:}

The patient data was collected anonymously from the electronic medical records using the prescribed data collection sheet. We included all patients who underwent AAD surgery except those who didn't have a post-operative CK/ Myoglobin value. We also excluded patients with renal dysfunction prior to surgery.

\section{Laboratory}

It is our department protocol to screen all cardiac surgery patients including aortic dissections with CK and myoglobin in the first day after surgery and to follow up these values if they are significantly high.

Patients were divided into two groups based on the collected data - Group 1- with RML (CK above cut off levels of $2500 \mathrm{U} / \mathrm{L}$ ) and Group 2 without RML. The determinants of RML and the impact of the same on the outcome - predominantly renal function was evaluated. Collected data included preoperative and intraoperative variable potentially affecting the renal outcome and incidence of RML: Age, body mass index, preoperative creatinine, Statin use, the delay from onset of symptoms to surgical intervention, renal artery involvement, duration of surgery, bypass time, cross-clamp time, circulatory arrest time, propofol dosage, etc. We also collected data relating to patient outcomes in terms of mortality and morbidity indicators -focusing mainly on the renal outcome.

\section{Study objectives}

The primary objective of this study was to elucidate the incidence of rhabdomyolysis following type A aortic dissection surgeries and to correlate it's the severity with the patient outcome -primarily in terms of renal function. Other outcome measures included mechanical ventilation duration, length of ICU stay, duration of hospital stay, and mortality. We also proposed to formulate a risk scoring system based on preoperative and intraoperative variables to predict the development of RML after AAD surgery

Study definitions:

We followed the same definition for Rhabdomyolysis which we used in our previous study.

In cardiac surgery, a higher cut off value $(2500 \mathrm{U} / \mathrm{L})$ to diagnose RML is proposed to account for the release of CK from related myocardial injury ${ }^{1}$. Patients were divided into two groups based on this diagnostic cutoff; Group A with RML (CK value above 2500 U/L) \& Group B without RML (CK below 2500 U/L). Acute kidney injury was defined using the KDIGO criteria AKI - KDIGO criteria define AKI as a $0.3 \mathrm{mg} / \mathrm{dl}$ ([?]26.5 $\mu \mathrm{mol} / \mathrm{l}$ ) Serum Creatinine increase from baseline within 48 hours of surgery, a 1.5 times Serum Creatinine increase from baseline within 7 days of surgery ${ }^{17}$. The original KDIGO criteria also use urine output below $0.5 \mathrm{ml} / \mathrm{kg} /$ hour for 6 hours to define AKI. Urine output criteria were not used to define AKI in our study due to data collection difficulties, as adopted from a similar study on the subject ${ }^{9}$.

\subsection{Statistical analysis}

Descriptive statistics are performed in the form of mean and standard deviations for continuous variables and frequency and percentages for categorical variables. Chi-square tests are performed to see the association between RML vs Non-RML group and other independent categorical variables whereas, student t-tests (unpaired) are performed to see significant mean differences between RML vs Non-RML group with continuous variables. Levene's Test for Equality of Variances has been taken into consideration for p-value. Correlation is performed between Creatine kinase 2 and creatinine $\mu \mathrm{mol} /$ liter. P-value 0.05 (two-tailed) is considered for statistically significant level ${ }^{18}$. SPSS 22.0 statistical software is used for the analysis. Clinical and laboratory data were entered into a database (Microsoft Excel 97, Redmond, WA, USA) and statistical analyses were performed (SPSS Inc., Version 16. Chicago IL, USA).

Observation and results

Perioperative variables 
Forty-four patients had undergone aortic dissection surgery in our center during the period 2011-2017. Out of these, 11 patients either died on the table or didn't have post-op CK levels and hence were excluded from the study. Of the remaining 33 patients, 21 patients (63.64\%) developed RML based on our diagnostic cut off value of CK (Group RML) and 12 did not (Group non-RML).

Other preoperative and intraoperative factors like critical preoperative states, congestive heart failure, renal artery involvement, cannulation method, type of surgical procedure etc. did not have a significant impact on the incidence of rhabdomyolysis postoperatively (Table 1). Patients who developed rhabdomyolysis had a higher body mass index (BMI) when compared to those who didn't, but this difference failed to achieve a statistical significance (P-value 0.07). Of note, patient with a delayed presentation for surgery tends to develop RML less frequently than those presenting early (P-value 0.03 )

\section{Outcome measures:}

There were 2 ICU mortalities in the RML group while there was none in the non-RML group, though the numbers were too small to achieve statistical significance. Acute kidney injury as defined by KDIGO criteria occurred in 22 patients out of 33 study patients ( $67 \%$ patients). There was a significantly higher incidence of AKI in the RML group (90\%) than the non-RML group (25\%). The cumulative occurrence of new postoperative dialysis was $12 \%$ in the study population and was observed only in the RML group (19\% within the group). Apart from significantly higher troponin levels in the RML group (P-value 0.003); the other morbidity parameters like length of ventilation, inotrope duration, ICU and hospital length of stay didn't have a statistically significant difference between the groups. (Table 2).

Figure 1 depicts the correlation between the change in creatinine (the difference between the peak creatinine and preoperative baseline) and CK levels. A significant correlation with a Pearson coefficient of 0.38 and P-value 0.0001 exists between the two.

Similarly, there was a significant correlation between the peak troponin levels and change in creatinine as outlined in figure 2

Discussion

The salient findings of this study were 1) Unusually high prevalence of RML amongst patients with AAD compared to other cardiac surgical patient population 2) Strong association of RML with AKI 3) Patients with high BMI were more involved in RML( even though the association didn't reach statistical significance ) 4) Delayed presentation for surgery was associated with a lesser risk of rhabdomyolysis. To the best of our knowledge no previous studies looked at these associations in Aortic Dissection Surgery.

Our study substantiates the hypothesis that aortic dissection surgery is associated with an unusually high incidence of RML (63\%) when compared to cardiac surgical cases in general reported by us before $(8.41 \%)^{1}$. The possible explanation for this predisposition could be occult ischemia to the lower limb following femoral cannulation, ischemia to paraspinal muscles due to malperfusion, prolonged positioning due to comparatively longer duration of the surgery ${ }^{1,15}$. It is postulated that the lumbar arteries that supply the paraspinal muscles may become compromised because of either hypoperfusion or occlusion from aortic cross-clamping, or ligation of the vessels within the false lumen of the dissection, or from athero-embolic phenomena. The resultant ischemia causes edema and necrosis of the paraspinal muscles, subsequently increasing the pressure within the paraspinal compartment ${ }^{15}$

Miller III et al. in an observational trial of 109 patients requiring thoracic/thoraco-abdominal aortic repair reported a dialysis requirement of $38 \%$ in the postoperative period. The dialysis rate was high in this study because the liberal inclusion criteria used for the same. Myoglobin levels were strongly predictive of postoperative renal dysfunction which was in agreement with our observations as well. But this study was done in patients undergoing thoraco-abdominal aortic aneurysm surgery without the use of cardiopulmonary bypass. Still, risk factors like femoral cannulation and prolonged positioning associated muscle damage is apparently common to both the patient $\operatorname{cohorts}^{16}$. The same group have also reported the relationship 
between loss of Somato-Sensory Evoked Potential signals in the cannulated leg and adverse renal outcome indicating leg ischemia as a potential contributing factor for $\mathrm{RML}^{19}$

The proposed risk factors for RML like the presence of diabetes or hypertension ${ }^{3}$ didn't have a significant impact on the incidence of RML in our study. Femoral cannulation could theoretically be associated with a higher incidence of rhabdomyolysis because of the potential for limb ischemia, but our study couldn't demonstrate a difference in outcome in terms of RML with femoral cannulation.

Patients who developed RML were more obese compared to the non-RML group, but this difference failed to achieve statistical significance. Zhao et al. (2015) reported a higher incidence of AKI (66.7\%) among obese patients with type A aortic dissection ${ }^{8}$. They found elevated preoperative serum Creatinine level and 72-h drainage volume as independent predictors of AKI, but they didn't look into the contribution of rhabdomyolysis to the development of kidney injury. The association between BMI and risk of RML has been well documented in bariatric and trauma surgeries as well ${ }^{20,21}$.

\section{Renal outcome:}

The incidence of AKI in our study population was $67 \%$, which is slightly higher than what is reported in the literature for AAD cases. It is noteworthy to mention though that incidence of new postoperative dialysis $(12 \%)$ in our study is comparable to the previous literature ${ }^{10-12}$. This difference could be attributed to the variation in the definition of AKI used in various studies, surgical technique, demographic characteristics, and institutional protocols for the initiation of CVVHD. Ko et al (2015) in their study which included 375 patients reported an incidence of $44.0 \%$ AKI out of which $9 \%$ required temporary dialysis and further $3 \%$ progressed to end-stage renal disease ${ }^{10}$. They also observed that the mortality and major adverse cardiovascular and cerebrovascular events correlated significantly with the severity of AKI. Extracorporeal circulation time, body mass index, perioperative peak serum C-reactive protein concentration, renal malperfusion, and perioperative sepsis were found to be risk factors for AKI.

Imasaka et al. (2015) in their retrospective review reported an incidence of $15.8 \%$ of renal replacement therapy. The proposed risk factors for postoperative renal replacement therapy were estimated glomerular filtration rate, coronary ischemic time, and total arch replacement ${ }^{11}$. Sansone et al. (2015) observed a 37.8\% incidence of AKI needing CVVHD after type A aortic dissection. Preoperative oliguria, longer Cardiopulmonary bypass / circulatory arrest times, and postoperative bleeding requiring a surgical revision were implicated as the predominant risk factors for the adverse outcome ${ }^{12}$. Similarly, Ghincea et al. (2019) reported a $32 \%$ incidence of AKI following aortic arch surgery. In the multivariable analysis, significant predictors of AKI were history of hypertension and CPB duration ${ }^{13}$. Kato et al. (2016) reported hypertension, type A aortic dissection, and low platelet levels as risk factors for AKI after aortic dissection surgery ${ }^{22}$,

\section{RML and high cardiac markers:}

One interesting observation from the study was that despite using a higher cut off value for CK to define RML (to account for the myocardial injury associated with cardiac surgery); we observed a significantly higher value of troponin in the RML group. This was not associated with other signs of myocardial damage as evidenced by an insignificant difference in inotropic requirements (Table 2). This difference could be explained by the fact that in RML, cardiac enzymes may be elevated unrelated to the degree of muscle damage ${ }^{23.24,25}$. Yet another explanation could be the higher incidence of AKI in the RML group. AKI is known to be associated with delayed clearance of troponins as reported in the previous study from our center $^{26}$.

\section{Relation between delay in presentation and severity of Rhabdomyolysis:}

Yet another finding which evolved from the review was that RML was more frequent in patients who were operated early in comparison to those intervened late. Only possible explanation could be some kind of stabilization of perfusion as the disease progresses towards chronicity. However we were not able to find any literature justifying this argument. 


\section{Preventive strategies:}

Interventions like aggressive fluid loading and forced diuresis titrated to a urine output of $200-300 \mathrm{ml} /$ hour might help to mitigate the severity of renal failure in patients at risk of $\mathrm{RML}^{4}$. Being a retrospective analysis, we were not in a position to assess the impact of these interventions on the renal outcome. However, in light of findings from our prior publication ${ }^{1}$, we are focusing on the above line of management in patients at risk of RML, especially when there is unexplained hyperkalemia in the immediate post-operative period.

\section{Conclusions}

In this study, we found high incidence of RML after aortic dissection surgery which was paralleled with an adverse renal outcome. Further large-scale prospective trials are warranted to investigate the predisposing factors and influence of RML on major morbidity and mortality outcomes.

\section{Study limitations:}

This study has several limitations- The sample size was small. The study was retrospective which precluded further standardization of design. The study was unable to identify the perioperative risk factors that predispose to the development of RML in aortic dissection surgery given its small sample size.

Definitions and abbreviations

ASA -American Society of Anesthesiologists

AAD-Ascending Aortic Dissection

ARF- Acute Renal Failure

AKI-Acute Kidney Injury- a $0.3 \mathrm{mg} / \mathrm{dl}$ ([?]26.5 umol/l) Serum Creatinine increase from baseline within 48 hours of surgery, a 1.5 times Serum Creatinine increase from baseline within 7 days of surgery

CVVHD- Continuous Veno Venous Hemodialysis

CK - Creatine Kinase

RML- Rhabdomyolysis - A fivefold elevation of serum CK levels above baseline ( > $2500 \mathrm{U} / \mathrm{L})^{1,3,4}$

\section{Declarations:}

\section{Ethics approval and consent}

This retrospective data review was approved by the medical research center of Hamad Medical Corporation, Doha, Qatar. The study was approved by Corporate Institutional review board with reference number -MRC01-17-058. Being a retrospective review, a waiver of informed consent was granted by the ethics committee. However, the Patient data were deidentified and then stored anonymously using computer generated codes.

\section{Consent for publication}

Not applicable.

\section{Availability of data and materials}

The manuscript related data and materials are available in Hamad Medical Corporation Medical Research Center database. Subject to approval from the medical research center of Hamad Medical Corporation, Data will be made available on request to the corresponding author

\section{Competing interest}

The authors have no conflicting interests to declare in relation to this manuscript

\section{Funding}

The study was funded by the Research Committee of Hamad Medical Corporation 


\section{Author Contribution:}

MK, study design, data collection, writing, reviewing, ASO: study design, contribution to concept, writing the manuscript and revising the final form. PS: data management and manuscript revision, data collection, interpretation of data, and revising of the manuscript, SS: data collection and revision. SH: writing and discussion management. SH: data collection and manuscript revision; HO data collection, RS: statistical analysis.

All authors read and approved the final manuscript.

Acknowledgment

The authors are immensely indebted to all members of the Hamad Medical Corporation for fulfilling the requirements for research. The authors thank all members of the Cardiothoracic surgery department, Cardiac anesthesia \& CTICU division, Heart Hospital, Hamad Medical Corporation, Qatar, for the required data and patient pool. The authors also thank each and every member from the medical research department of Hamad Medical Corporation for their support throughout this project. Our sincere thanks to Dr. Abulaziz Alkhulaifi, Chairman of the Cardiothoracic Surgery Department for his administrative support.

References

1. Omar AS., Ewila H, Aboulnaga S et al. Rhabdomyolysis following cardiac surgery: a prospective, descriptive, single-center study. BioMed research international, 2016; 2016:7497936.

2. Shapiro ML, Baldea A, Luchette FA. Rhabdomyolysis in the intensive care unit. J Intensive Care Med $2012 ; 27: 335-342$

3. Warren JD, Blumbergs PC, Thompson PD. Rhabdomyolysis: a review. Muscle Nerve 2002; 25:332.

4. Cote, D.R., Fuentes, E., Elsayes, A.H. et al. A "crush" course on rhabdomyolysis: risk stratification and clinical management update for the perioperative clinician. J Anesth 34, 585-598 (2020)

5. Hofmann D, Buettner M, Rissner F et al.. Prognostic value of serum myoglobin in patients after cardiac surgery. J Anesth. 2007;21(3):304-10. Epub 2007 Aug 1.

6. Benedetto U, Angeloni E, Luciani R et al. Acute kidney injury after coronary artery bypass grafting: does rhabdomyolysis play a role? J Thorac Cardiovasc Surg. 2010 Aug;140(2):464-70. Epub 2010 Apr 22.

7. O'Neal JB, Shaw AD, Billings FT. Acute kidney injury following cardiac surgery: current understanding and future directions. Crit Care 2016; 20(1):187

8. Zhao H, Pan X, Gong Z, et al. Risk factors for acute kidney injury in overweight patients with acute type A aortic dissection: a retrospective study. J Thorac Dis. 2015;7:1385-1390

9. Mussa FF, Horton JD, Moridzadeh R et al.. Acute Aortic Dissection and Intramural Hematoma, A Systematic Review. JAMA. 2016;316(7):754-763.

10. Ko T, Higashitani M, Sato A, et al. Impact of acute kidney injury on early to long-term outcomes in patients who underwent surgery for type A acute aortic dissection.Am J Cardiol. 2015;116:463-468

11. Imasaka K-i, Tayama E, Tomi ta Y. Preoperative renal function and surgical outcomes in patients with acute type A aortic dissection. Interact CardioVasc Thorac Surg 2015;20:470-6.

12. Sansone F, Morgante A, Ceresa F et al. Prognostic Implications of Acute Renal Failure after Surgery for Type A Acute Aortic Dissection. AORTA (Stamford) 2015 Jun 1;3(3):91-7

13. Ghincea CV, Reece TB, Eldeiry M et al. Predictors of acute kidney injury following aortic arch surgery. J Surg Res. 2019;242:40-46.

14. Qian SC, Ma WG, Pan XD et al. Renal malperfusion affects operative mortality rather than late death following acute type A aortic dissection repair. Asian Journal of Surgery. 2020 Jan 1;43(1):213-9.

15. Anthony DG, Diaz J, Allen BC et al. Occult rhabdomyolysis after acute type A aortic dissection. Critical Care Medicine.2011; 39(8):1992-1994.

16. Miller CC III, Villa MA, Sutton J et al. Serum myoglobin and renal morbidity and mortality following thoracic and thoraco-abdominal aortic repair: does rhabdomyolysis play a role? Eur J Vasc Endovasc Surg. 2009; 37:388-94. 
17. Khwaja A. KDIGO clinical practice guidelines for acute kidney injury. Nephron Clin Pract. 2012;120:c179- 84

18. Munro BH. Statistical methods for health care research fourth edition. University of Pennsilvania: Boston collage Lipincott U.S.A; 2001. p. 1-412.

19. Miller III CC, Villa MA, Achouh P, et al. Intraoperative skeletal muscle ischemia contributes to risk of renal dysfunction following thoracoabdominal aortic repair. European Journal of Cardiothoracic Surgery 2008;33:691e4.

20. Youssef T, Abd-Elaal L, Zakaria G et al. Bariatric surgery: rhabdomyolysis after open Roux-en-Y gastric bypass: a prospective study. Int J Surg. 2010;8(6):484-8.

21. Chan JL, Imai T, Barmparas G et al. Rhabdomyolysis in obese trauma patients. The American Surgeon. 2014 Oct 1;80(10):1012-7.

22. Kato A, Ito E, Kamegai N, Mizutani M et al. Risk factors for acute kidney injury after initial acute aortic dissection and their effect on long-term mortality. Ren Replace Ther. 2016;2(1):53

23. Punukollu G, Gowda RM, Khan IA, et al. Elevated serum cardiac troponin I in rhabdomyolysis. Int J Cardiol. 2004;96(1):35-40.

24. Inbar R, Shoenfeld Y. Elevated cardiac troponins: the ultimate marker for myocardial necrosis, but not without a differential diagnosis. Isr Med Assoc J. 2009;11(1):50-53.

25. Li SF, Zapata J, Tillem E. The prevalence of false-positive cardiac troponin I in ED patients with rhabdomyolysis. Am J Emerg Med . 2005;23(7):860-863

26. Omar AS, Mahmoud K, Hanoura S, et al. Acute kidney injury induces high-sensitivity troponin measurement changes after cardiac surgery. BMC Anesthesiol. 2017;17(1):15.

\section{Hosted file}

figure (1).docx available at https://authorea.com/users/421166/articles/527299-incidenceand-outcome-of-rhabdomyolysis-after-type-a-aortic-dissection-surgery-a-retrospectiveanalysis

\section{Hosted file}

figure (2).docx available at https://authorea.com/users/421166/articles/527299-incidenceand-outcome-of-rhabdomyolysis-after-type-a-aortic-dissection-surgery-a-retrospectiveanalysis

\section{Hosted file}

table 1.docx available at https://authorea.com/users/421166/articles/527299-incidenceand-outcome-of-rhabdomyolysis-after-type-a-aortic-dissection-surgery-a-retrospectiveanalysis

\section{Hosted file}

table 2.docx available at https://authorea.com/users/421166/articles/527299-incidenceand-outcome-of-rhabdomyolysis-after-type-a-aortic-dissection-surgery-a-retrospectiveanalysis 